\title{
El gol como unidad de medida de rendimiento en futsal \\ Goal as a performance measurement unit in futsal
}

Javier Alvarez Medina, Javier Ramírez San Jose, Victor Murillo Lorente

Universidad de Zaragoza (España)

\begin{abstract}
Resumen. Introducción: El fútbol sala es un deporte moderno que se caracteriza por ser un juego muy rápido con veloces transiciones que generan continuas ocasiones de gol. Los equipos tanto a nivel nacional como internacional cada vez están más igualados por lo que el análisis y comprensión de lo que ocurre en la competición cada vez es más importante para poder tener una ventaja en la competición. El objetivo de este trabajo es analizar y comparar los goles como unidad de medida acontecidos en tres de las mejores ligas europeas masculinas durante la temporada 2014-2015, definir sus características y establecer las semejanzas y diferencias entre ellas. Método: La muestra asciende a la totalidad de la población objeto de estudio, que corresponde a 110 partidos en la liga italiana con un total de 11 equipos, 240 en la liga española con 16 equipos y 273 en la liga rusa con 14 equipos, lo que supone un total de 623 partidos de los que se han podido analizar 619 y una muestra ( $n$ ) alcanzada de 4716 goles. Resultados y conclusiones: No se han encontrado diferencias significativas entre la capacidad goleadora de las diferentes ligas estudiadas, siendo la liga rusa la más goleadora con una media por partido de 8.16 goles seguida por la española con 7.37 goles y la italiana con 6.67 goles. Los equipos locales anotan el $54.2 \%$ de los goles por el $45.8 \%$ como visitante. No se han encontrado diferencias significativas entre las ligas.
\end{abstract}

Palabras clave: deporte, futsal, gol, ligas europeas, rendimiento.

\begin{abstract}
Introduction: Futsal is a modern sport characterized by a very fast game with speed transitions generating continuous scoring chances. National and international teams have very similar levels of performance, therefore the analysis and understanding of competition events become increasingly important in order to obtain an advantage in competition. The objective of this work is to analyze and compare goals as measurement units in three of the best male European leagues during the 2014-2015 season; to define their characteristics; and to establish the similarities and differences between them. Method: The sample includes the entire population under study, which corresponds to 110 matches in the Italian league with a total of 11 teams, 240 in the Spanish league with 16 teams, and 273 in the Russian league with 14 teams, which account for a total of 623 matches, 619 of which have been analyzed, providing a total of 4,716 goals. Results and conclusions: No differences were found between the scoring capacity of the different leagues studied, teams in the Russian league scoring the highest amount of goals per game (8.16), followed by the Spanish (7.37), and the Italian (6.67). Home teams score 54.2\% of the total goals, whereas away teams account for $45.8 \%$ of the total. No significant differences were found between the leagues.
\end{abstract}

Keywords: sport, futsal, goal, European leagues, performance.

\section{Introducción}

El objetivo de los deportes de equipo es conseguir el mayor número de goles o puntos, ya que la finalización de las acciones ofensivas con éxito marcan el resultado final (Lago, Cancela, López, Fernández \& Veiga, 2006). Dentro de estos deportes encontramos modalidades con diferencias y semejanzas que vienen determinadas principalmente por el reglamento. Así, el fútbol sala y el balonmano, a pesar de sus diferencias evidentes donde uno se juega fundamentalmente con los pies y otro con las manos, son los únicos que comparten el mismo terreno de juego ( 40 x 20 metros) y reglas parecidas que permiten desde realizar un número ilimitado de cambios, a utilizar un jugador de campo como portero, lo que hace que tengan muchas semejanzas en su estilo de juego (Beiztegui-Casado, Oliver-Coronado \& Sosa-González, en prensa; Méndez-Dominguez, Gómez-Ruano, Ruiz-Pérez, \& Cul, 2017; Saavedra, Porgeirsson, Kristjánsdóttir, Chang \& Halldórsson, 2017). Estas modalidades se identifican con esfuerzos de tipo intermitentes de gran intensidad, con recuperaciones activas incompletas durante todo el partido, predominando un juego muy rápido con constantes transiciones ataque-defensa y generando numerosas ocasiones para alcanzar el objetivo del juego «el gol» (Agras, Ferragut \& Abraldes, 2016; Gómez, Méndez, Indaburu \&

Fecha recepción: 25-09-18. Fecha de aceptación: 21-02-19

Victor Murillo Lorente

victormurillo1301@hotmail.com
Travassos, 2018; Pascual-Verdú, Álvarez-Amorós, CarbonellMartínez \& Pérez-Turpin, 2019; Saavedra, et al., 2017; Schneider, Da Cunha-Voser \& Gomes-Voser, 2015). En contraposición, el fútbol, a pesar de las similitudes aparentes con el futsal, tiene grandes diferencias debido principalmente a las grandes dimensiones del terreno de juego que se pueden concretar en la escasez de ocasiones de gol, hasta el punto que se puede ganar un partido en una única finalización y en cualquier momento del partido (López-García, Maneiro-Dios, Ardá-Suárez, Rial-Boubeta, Losada-López \& Casal-Sanjurjo, 2018; Pascual, et al., 2019).

En todos los deportes de equipo, el gol, el tanteo, su índice de aprovechamiento, su análisis y cómo se produce es el indicador y predictor más importante del rendimiento (Agras, et al., 2016; Álvarez, Murillo \& García, 2018; Gómez, et al., 2018; Scheider, et al., 2015), y por ello el objetivo principal de las investigaciones científicas, donde el futsal tiene un déficit con respecto a otras modalidades de equipo como el fútbol, baloncesto, balonmano, waterpolo siendo necesarios más estudios que permitan comprender mejor todo lo que ocurre en la competición (Moore, Bullough, Goldsmith \& Edmondson, 2014; Agras, et al., 2016; Gómez, et al., 2018). Agras et al. 2016 en una revisión sistemática realizada entre abril 1993 y diciembre 2014 sobre el análisis de los partidos en futsal, concluye que solo el $13 \%$ de las publicaciones sobre el tema se realizan en revistas científicas.

La Liga española y su selección nacional están consideradas como una de las mejores del mundo, lo que se ratifica en el ranking oficial tanto de la Federación Internacional de Asociaciones de Fútbol (FIFA) y la Unión 
Europea de Asociaciones de Fútbol (UEFA) donde ocupan los primeros puestos (estadísticas FIFA, 2016). Esto junto a la actual expansión, profesionalización y globalización del futsal a nivel mundial, con más de treinta millones de practicantes en el mundo en el 2012 (Agras, et al., 2016), ha hecho que España sea un país exportador tanto de jugadores como de técnicos por todos los mercados emergentes del mundo. Así, la mayoría de los estudios existentes de futsal de campeonatos de larga duración tienen como muestra la liga brasileña y española, estableciendo un rango de 4.455.01goles/partido para la liga brasileña (Cassita, 2015; Da Cuna, Goncalves \& Eloi, 2016) y de 6.44-7.15 goles/partido para la liga española (Álvarez, et al., 2018; Giani, Faria \& Aparecida da Silva, 2018). No existiendo estudios comparativos de las principales ligas europeas surge la pregunta de esta investigación ¿̇las principales ligas europeas se comportan como la española o tienen sus propias características diferenciadoras?

El objetivo de este trabajo es analizar y comparar los goles como unidad de medida acontecidos en tres de las mejores ligas europeas masculinas, definir sus características y establecer las semejanzas y diferencias entre ellas, con respecto a la más estudiada como es la española.

\section{Material y método}

Estudio descriptivo, cuantitativo, comparativo y prospectivo de todos los goles de tres de las mejores ligas profesionales europeas de fútbol sala según títulos europeos: española, italiana y rusa.

La muestra asciende a la totalidad de la población objeto de estudio, que corresponde a 110 partidos en la liga italiana con un total de 11 equipos, 240 en la liga española con 16 equipos y 273 en la liga rusa con 14 equipos, lo que supone un total de 623 partidos, de los que se han podido analizar 619 y una muestra $(n)$ alcanzada de 4716 goles de la temporada 2014-2015 durante su fase regular.

La recogida de datos se hizo a través de las actas finales de los partidos. Se pidió permiso a las respectivas ligas por escrito para el acceso a las mismas, el cual fue concedido y remitidos a la web oficial donde están publicadas las mismas, siendo el acceso público a los datos registrados: tarjetas amarillas, tarjetas rojas, goles, minuto del gol, jugadores titulares, convocados, entrenadores, árbitros, sede donde se disputa el partido y espectadores.

\section{Justificación de la población a estudio}

Se han elegido las ligas española, rusa e italiana por considerar que son la mejor referencia para estudiar el objeto de estudio al ser, en el momento de la toma de datos, las mejores según títulos conseguidos en los campeonatos europeos organizados por la UEFA entre 1996-2015, España con siete títulos, Italia con dos y Rusia con uno (Göral, 2018)

\footnotetext{
Variables a estudio

Goles anotados por:

- Temporada, vuelta, jornada (y ronda Rusa)

- Partido

- Equipo

- Jugando como local y visitante
}

\section{- Clasificación final}

\section{Instrumentos de análisis de datos}

Paquete estadístico SPSS, versión 22.0, para la estadística descriptiva e inferencial en la búsqueda de relaciones asociativas entre variables categóricas. Datos descriptivos dados en frecuencias, medias y porcentajes y estadística inferencial a través de tablas de contingencia utilizando las pruebas paramétrica y no paramétricas Chi-cuadrado y Rho de Spearman para establecer la relación entre variables estableciendo significación estadística cuando el valor es Â.05.

\section{Resultados}

\section{Número de goles por temporada-jornada-partido- equipo}

Rusia obtiene el 47.2\% de los goles (2226), seguido por España con 37.2\% (1755) e Italia con 15.6\% (735).

La media global de goles es de 49.84 por jornada, 7.40 por partido y 3.60 por equipo.

La liga Española tiene una media por jornada de 58.97 goles, por partido de 7.37 y por equipo de 3.69.

La liga Rusa tiene una media por jornada de 57.18 goles, por partido de 8.16 y por equipo de 4.08. En el caso de la liga Rusa la jornada es doble en 13 ocasiones, por lo que se ha hallado la media de jornada por los partidos disputados de cada equipo.

La liga Italiana tiene una media por jornada de 33.36 goles, por partido de 6.67 y por equipo de 3.03 .

\section{Número de goles por vuelta}

Se observan valores muy semejantes entre todas ellas, siendo el porcentaje más alto en la segunda vuelta en todas las ligas, sin encontrar diferencias significativas.

\section{Número de goles jugando como local o visitante}

Los resultados muestran que como local se anotan el $54.2 \%$ de los goles (2554) y como visitante el $45.8 \%$ (2162).

El mayor porcentaje de goles en todas las ligas se da como local, siendo un 54.8\%, 52.8\% y 56.9\%, para España, Rusia e Italia respectivamente y como visitante España el $45.2 \%$, Rusia $47.2 \%$ e Italia el $43.1 \%$. No se han encontrado diferencias significativas entre las ligas.

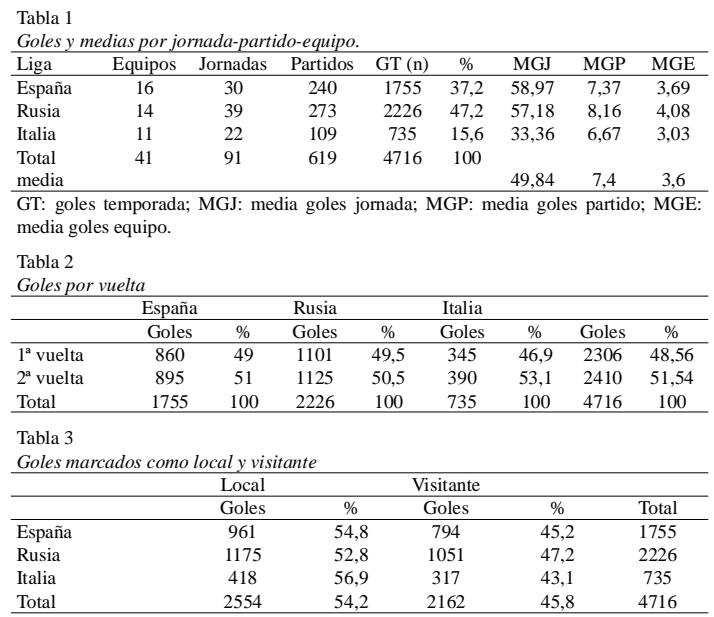




\section{Número de goles y clasificación final}

La correlación a través de la Rho de Spearman ha dado unos valores de:

- Goles anotados y la clasificación final ofrece un valor significativo de .037 en los goles a favor y una correlación inversa de -.327.

- Goles en contra se encuentra una significatividad de .000 y una correlación directa con valor .626.

- Diferencia de goles y clasificación final ofrece una significatividad de 0.000 y una correlación inversa con valor -.912 .

\section{Discusión}

\section{Número de goles por temporada jornada-partido- equipo}

Para explicar las diferencias encontradas en los goles totales por temporada y por jornada se debe tener en cuenta las particularidades de cada competición, siendo distintos el número de partidos jugados, así como el número de equipos participantes y por tanto jornadas disputadas.

Respecto a los goles totales por temporada en valores absolutos Rusia aporta casi la mitad de los goles con 2226, lo que es el $47.2 \%$ de los goles, seguido por España con 1755 y un $37.2 \%$ e Italia con 735 y un 15.6\%. Las diferencias encontradas se deben fundamentalmente a la diferencia de partidos jugados: 273 en Rusia, 240 en España y 109 en Italia.

Con respecto a goles/jornada se obtiene una media de 49.84 goles, siendo España la que obtiene la media más alta con 58.97 goles, seguida por Rusia con 57.18 goles y por último Italia con 33.36 goles. Las diferencias encontradas se deben fundamentalmente al distinto número de equipos participantes, siendo España la mayor con 16, Rusia con 14 e Italia con 11.

Los resultados globales dan una media de 7.4 goles/ partido y de 3.6 goles/equipo. Rusia obtiene la mayor media con 8.16 goles/partido y 4.08 goles/equipo; seguida por la liga española con 7.37 goles/partido y 3.69 goles/partido; y la italiana con un 6.67 goles/partido y 3.03 goles/equipo. No se han encontrado diferencias significativas entre las ligas. Grela (2012) obtiene valores muy similares entre las temporadas 2008 a la 2012 de la liga española con un rango 3.49-3.62 goles por goles/equipo y Giani et al. (2018) de la temporada 2015-2016 de la Liga Española con una media de 7.15 goles/partido.

Para entender mejor estos resultados se debe saber que el futsal sufrió un cambio de normativa en el año 2006 con el objetivo de unificar los reglamentos a nivel mundial de FIFA y UEFA. Las modificaciones de los reglamentos en los deportes de equipo suelen buscar fomentar un juego más rápido y dinámico, que genere una mayor consecución de puntos o goles, para que sea más atractivo al espectador y se ajuste a las necesidades, generalmente, televisivas (Álvarez, Murillo \& García, 2018; Cachón, Campoy, Linares, Rodrigo \& Zagalaz, 2012; Cachón, Valdivia, Lara, Zagalaz \& Berdejo, 2014; Pascual, et al., 2019). Estos cambios modifican la lógica interna del juego, como les ha pasado a otras modalidades de equipo como Waterpolo (García-Marín, Argudo-Iturriaga \& Alonso-Roque, 2015; Iglesias-Pérez, García-Ordóñez \& Touriño-González, 2018; Sabio-Lago,
Guerra-Balic, Cabedo-Sanromà, Solà-Santesmases \& Argudo-Iturriaga, 2018).), balonmano (Beiztegui, et al., en prensa; Jimenez-Olmedo, Espina-Agullo \& Manchado, 2017), baloncesto (Pérez-Ferreirós, Kalen \& Rey, 2018).), vóley (GilArias, Claver-Rabaz, Fernández-Echeverría, MorenoDomínguez \& Moreno-Arroyo, 2016)), hockey patines (Trabal-Tañá, Daza \& Riera, 2019), ya que el reglamento establece la estructura formal, lo que está permitido y lo que no y le imprime un carácter propio a la modalidad deportiva (Álvarez, et al., 2018a; Vizcaíno, Sáenz-López \& Rebollo, 2016)

Cachón et al. (2014) concluye en su estudio «análisis cualitativo del cambio de reglas de juego del fútbol sala (2006) en España», basado en la opinión de jugadores, entrenadores y directivos a través de un cuestionario realizado en la XX edición del Campeonato de la Copa de la Liga Nacional de Futsal en 2009 que la nueva normativa de futsal afectó disminuyendo el espectáculo, en especial en lo relativo a la vistosidad (técnica y táctica), al número de goles y a la velocidad de juego, posteriormente esto es ratificado por otros autores como Méndez et al. (2017), Pascual et al. (2019) y Álvarez et al. (2018a) que compara los goles antes del cambio de reglamento en la temporada 2002-2003, donde se consiguieron 1927 goles, con una media por equipo de $120.38 \pm 28.58$ y por partido de 4 goles/equipo y en la temporada 2013-2014, después del cambio, donde se consiguieron 1355 goles, con una media por equipo de $90.40 \pm 27.72$ goles y por partido de 3.22 goles/equipo, existiendo un descenso de la media de goles por partido con diferencias estadísticamente significativas $(\mathrm{p}=.004)$. Para Álvarez et al. (2018a) las modificaciones del reglamento modificaron la forma de juego afectando a su lógica interna y reduciendo significativamente el número de goles. Las principales variaciones fueron según variable tipo de jugada:

- Se pasó de un $24 \%$ de goles debido a robo a un $9.9 \%$. Antes del cambio de normativa el saque de portero se tenía que hacer de manera que el balón botara o tocara a un jugador en la pista propia, lo que hacía que la presión en pista contraria tuviera sentido. El cambio de normativa posibilita que el portero saque a pista contraria directamente, lo que elimina prácticamente las tácticas de presión en pista contraria, favoreciendo un juego más estático, con menos movimiento y menos espectacular.

- Aumentan los goles conseguidos por contraataques pasando de un $15 \%$ a un $21.3 \%$ en la temporada 20013-2014. Ante la posibilidad de un saque directo del portero el repliegue defensivo es mucho mayor, provocando defensas atrasadas, lo que genera un ataque ante una defensa estructurada que si roba el balón genera el contraataque y la superioridad numérica.

Según la variable estrategia se pasó de un 34\% del total de goles a un $22 \%$, donde los valores absolutos descienden muy significativamente en todas las categorías, excepto en el penalti.

- Con respecto al córner y saque de banda: se mantiene sobre el 55\% de los goles de estrategia pero los valores absolutos bajan a la mitad confirmando la dificultad que tiene el cambio de saque con la mano al pie para la consecución de goles. Estos datos ratifican lo expresado por Cachón et al. (2012) en cuanto a la reducción de goles en jugadas de estrategia y que antes se podía manejar una mayor riqueza 
de acciones a balón parado y por Pascual et al. (2019).

-En cuanto a las faltas y doble penalti: hay un descenso en valores absolutos de los goles en estas acciones como consecuencia del cambio en la forma de defender el saque del portero. Las defensas se retrasan y desaparece la presión intensiva en prácticamente toda la pista, lo que reduce el número de faltas que se comenten y por lo tanto el número de doble penaltis y de goles marcados en estas acciones.

Los resultados del presente estudio parecen indicar una recuperación paulatina de los goles con una media de 7.31 goles/partido y de 3.69 goles/equipo, si bien no llegan a las medias anteriores al cambio normativo.

Los valores obtenidos por la liga rusa son los más altos con una media de 8.16 goles/partido y 4.08 goles/equipo y se encuentran en los valores anteriores al cambio normativo de la liga española confirmando ser la liga donde más goles y mayores medias se obtienen.

Este mayor número de goles de la liga rusa con respecto a la italiana y la española puede ser debido al poder económico de las mismas. Generalmente, los jugadores más cotizados son los que tienen más cualidades ofensivas, con una mayor eficiencia ofensiva. La calidad de los jugadores ofensivos que un equipo tiene en plantilla, va a determinar en gran medida los goles que se pueden anotar en un partido. Desde la crisis financiera europea del 2006, tanto la liga española como la italiana han padecido un recorte muy grande en sus presupuestos lo que ha hecho que grandes jugadores de sus ligas, muchos de ellos del continente sudamericano y concretamente brasileños, hayan preferido jugar en otros países que no se han visto tan afectados por la crisis como es el caso de la liga rusa.

Otra razón de la diferencia de goles puede ser la idea compartida entre los expertos deportivos, de que los rendimientos de los equipos a nivel nacional e internacional están cada vez más igualados, motivados seguramente por el efecto de la globalización y el estudio del rival (AbdelHakin, 2014), haciendo que la táctica, especialmente la defensiva, de los equipos esté mucho más trabajada disminuyendo la media de goles. En esta línea encaja el resultado de la liga italiana, con la media de goles por partido más baja de las ligas analizadas con 6.67 goles/ partido muy parecida a las obtenidas en los estudios de campeonatos de selecciones de corta duración donde las precauciones y estudios previos del rival son importantísimos ya que un error puede significar la eliminación de la competición. Así, en la Copa del Mundo de 2012 en Thailandia, en 52 partidos se consiguen 349 goles con una media de 6.71 goles/partido, en la de 2008 en Brasil, en 56 partidos se consiguieron 387 goles con una media de 6.91 goles/partido (Abdel-Hakin, 2014) y en el Campeonato de Europa realizado en Serbia 2016 se anotaron 129 goles, obteniendo una media de 6.45 goles/ partido (Göral, 2018).

Los datos obtenidos los podemos comparar con estudios de la liga brasileña, así en la temporada 2013 en la segunda vuelta se obtiene una media de 4.45 goles/partido (Cassita, 2015) y en la temporada 2014 de 5.01 goles/partido (Da Cuna, et al., 2016) valores inferiores a los de nuestro estudio, lo que puede indicar la elevada calidad ofensiva de las ligas europeas, gracias, entre otras cosas, a que consiguen contratar a muchos de los mejores jugadores de su continente y de su liga.

En los pocos estudios que analizan los goles de las ligas femeninas de futsal encontramos una media de 5.5 goles/ partido en las temporadas 2010 y 2011 de la liga brasileña (Massardi, Oliveira \& Navarro, 2011) y un poco más alta en la fase final del XVIII Campeonato Brasileño con una media de 6 goles/partido (Santana, Laudari, Istchuk Arruda, 2013).

Haciendo referencia a los resultados obtenidos se puede decir que la liga rusa aboga por un estilo de juego de mayor vistosidad donde prima lo ofensivo frente a lo defensivo, aspectos que autores como Cachón et al. (Cachón, et al., 2014) señalan importantes para la mayor diversión del público y por consiguiente mayor espectáculo.

\section{Número de goles por vuelta}

El 48.56\% de los goles se consiguen en la primera vuelta y el $51.54 \%$ en la segunda. No se encuentran diferencias significativas, siendo mayor en todas las ligas el porcentaje de goles marcados en la segunda vuelta que en la primera. Álvarez (2011) en el estudio del rendimiento deportivo de un equipo durante la temporada 2002-2003 de la liga profesional de fútbol sala en España, obtiene unos valores parecidos algo inferiores en la primera vuelta con un $43.79 \%$ y superior en la segunda con un $56.20 \%$.

\section{Número de goles jugando como local o visitante}

Como local se anotan el 54.2\% de los goles (2554), un $8.4 \%$ más que como visitante con un $45.8 \%$ (2162). El mayor porcentaje de goles en todas las ligas se da como local, no encontrando diferencias significativas entre ellas.

Grela (2013) referencia el total de goles por visitante y local sobre 5 temporadas de la liga profesional española, obteniendo un rango entre el 54.23-56.01\% los equipos locales, frente a un $43.99-45.77 \%$ los visitantes, resultados que coinciden con los obtenidos en este estudio.

Campos, Pellegrinotti, Pasquarelli, Rabelo, Santa Cruz y Gómez (2015) al estudiar tres temporadas 2012-2014 de la liga brasileña obtiene que los equipos locales anotaron un 25\% más de goles que los visitantes, valores superiores a los obtenidos en este trabajo que establece un 8.4\% más. Estas diferencias pueden deberse a las diferencias del estilo de juego en la liga brasileña que en las ligas europeas, lo que puede ser motivo de otro estudio para poder establecer si existen diferencias entre la liga brasileña y las europeas.

En una revisión sobre la ventaja de jugar en casa en el fútbol español (1998-2011) (Saavedra-García, GutiérrezAguilar, Fernández-Romero \& San Marques, 2015) se encuentran diferencias muy significativas entre los goles marcados como local que como visitante estableciendo que se marcan el doble como local que como visitante. Estos datos son de otra modalidad deportiva como el fútbol donde las finalizaciones a portería son mucho menores que en el futsal y se estima que solo un $1 \%$ de los disparos que se efectúan obtienen el gol (López, et al., 2018), muy diferente al futsal.

El mayor número de goles de los equipos locales puede explicarse por el llamado fenómeno de jugar en casa «home advantage» (HA). Un metaanálisis de Jamieson (2010) confirmó que las ventajas de jugar en casa existen en los deportes de equipo, presentando valores alrededor del 60\%. 
Los pocos estudios de futsal que lo analizan obtienen los mismos resultados, entre un 61-63\%, en la liga profesional de España y Brasil (Campos, et al., 2015; Martínez-Martínez \& González-García, 2019; Sampedro \& Prieto, 2011). El HA incluye factores situacionales como fatiga por el viaje y la presión ambiental para el equipo visitante y el apoyo del grupo, sensación de pertenencia, arbitrajes, conocimiento de la pista, etc. para el local. (Agnew \& Carron, 1994; Campos, et al., 2015; Nevill \& Holder, 1999; Nevill, Balmer \& Williams, 1999; Sutter \& Kocher, 2004). En este sentido, Wallace, Baumeister y Vohs (2005) cuando los equipos juegan en su propio campo, la multitud tiende a aumentar el rendimiento de los jugadores a través de su apoyo y los jugadores locales tienden a tener mayor confianza que los visitantes. La familiarización total con el entorno y la pista, tipo de suelo, los puntos de referencia como las líneas, vigas de color o monocromo, grandes o pequeñas áreas de salida, distancias a la valla son factores que generan confianza en el equipo local y pueden ayudar al rendimiento del equipo (Carré, Muir, Belanger \& Putnam, 2006).

Un estudio de las principales ligas europeas de fútbol establece que no hay diferencias significativas entre ellas al comparar el efecto de marcar primero y la localización del partido, concluyendo que son muy similares (Martínez, et al., 2019).

Se necesitan más estudios que analicen el efecto HA en mayor profundidad estudiando cómo afecta sobre el resultado final conseguir el primer gol, llegar con el marcador al descanso a favor, ya que sin duda estas variables afectarán al estado psicológico de los jugadores (Nevill et al., 1999a).

Con mayor razón el HA debería estudiarse en la liga rusa, ya que debido a sus peculiaridades geográficas, que conlleva recorrer grandes distancias para jugar los partidos, utilizan el sistema de doble ronda, lo que significa que el mismo fin de semana, en menos de cuarenta y ocho horas, se juegan dos partidos en el mismo escenario y contra el mismo equipo. Sería muy interesante saber cómo afecta el resultado del primer partido sobre el segundo y como afectan los factores situacionales como dormir, comer fuera de casa durante varios días.

Este sistema de doble ronda se utiliza en la liga española en el play off por el título de Liga por lo que puede ser igual de interesante para los equipos que opten a disputar el mismo.

\section{Número de goles y clasificación final}

Se relacionan los goles anotados, encajados y la diferencia entre ellos con la clasificación final, para lo que se aplicó la prueba estadística Rho de Spearman obteniendo una relación significativa en todos ellos.

Los resultados coinciden con las conclusiones expuestas por distintos autores que hablan de una relación directa entre posición en la tabla y número de goles anotados por el equipo. Álvarez, Puente, Manero y Manonelles (2004), analiza la relación entre la clasificación final de la temporada 2002-2003 de la liga nacional de fútbol sala con los goles anotados y obtiene que cinco de los equipos más goleadores acaban entre los ocho primeros, mientras que dos de los tres menos realizadores quedaron último y penúltimo en la clasificación. Concluyendo que existe una relación directa entre los goles anotados y la clasificación final. Resultados muy parecidos obtiene Giani et al., (2018) con la liga española de la temporada 2015-2016, donde siete de los equipos más goleadores acaban entre los ocho primeros y tres de los últimos cuatro clasificados son los que menos goles consiguen.

Kacem, Guemri, Naffeti \& Elloumi (2016) analizan el Campeonato del Mundo 2012 y la Eurocopa de 2014 concluyendo que los equipos que más goles meten son los que ganan, estableciendo las probabilidades de ganar en un $75 \%$ si se marcan 4 goles por el 5\% de perder.

Los resultados del presente estudio coinciden con los estudios anteriores, se obtiene un valor significativo de .037 en los goles a favor y la clasificación final y una correlación inversa de -.327. Siete de los equipos más goleadores ocupan un puesto en la clasificación final entre los ocho primeros. Esta estadística es todavía mayor para la liga italiana, donde el 100\% de los equipos más goleadores ocupan un puesto entre los ocho primeros. La liga rusa es la más variable con únicamente cinco de los equipos más goleadores entre los ocho primeros. Esta diferencia puede deberse a que juegan más jornadas y al alto número de goles con los que acaban la temporada.

Existe una relación clara entre los goles anotados por un equipo y la posición que dicho equipo ocupa en la tabla final, y esta relación se hace más evidente cuanto más en los extremos de la clasificación se posicionan (tabla 4).

\begin{tabular}{lccc}
$\begin{array}{l}\text { Tabla } 4 \\
\text { Clasificación por goles anotados }\end{array}$ & final liga Española. & \\
\hline Equipo & $\begin{array}{c}\text { Goles } \\
\text { anotados }\end{array}$ & $\begin{array}{c}\text { Clasificación goles } \\
\text { anotados }\end{array}$ & $\begin{array}{c}\text { Clasificación } \\
\text { final }\end{array}$ \\
\hline El Pozo Murcia & 152 & 1 & 3 \\
Movistar Inter FS & 151 & 2 & 1 \\
FC Barcelona Lassa & 145 & 3 & 2 \\
Catgas E. Santa Coloma & 140 & 4 & 12 \\
Palma Futsal & 122 & 5 & 5 \\
Montesinos Jumilla & 119 & 6 & 13 \\
Peñíscola Bodegas Dunviro & 116 & 7 & 8 \\
Magna Gurpea & 115 & 8 & 6 \\
Jaén Paraíso Interior & 113 & 9 & 4 \\
Aspil Vidal Ribera Navarra & 105 & 10 & 7 \\
DLink Zaragoza & 105 & 11 & 10 \\
Burela Pescados Rubén & 97 & 12 & 9 \\
Santiago Futsal & 83 & 13 & 11 \\
Levante UD DM & 75 & 14 & 14 \\
Uruguay Tenerife & 72 & 15 & 15 \\
Prone Lugo FS & 59 & 16 & 16 \\
\hline
\end{tabular}

El poder financiero de los primeros equipos de la clasificación y la calidad de los jugadores que tienen en sus plantillas hace posible en ocasiones que sus registros goleadores sean superiores. Sin embargo, se observa que en la zona central de la clasificación las diferencias con respecto a este criterio son más cambiantes indicando que existe una gran igualdad entre los equipos de mitad de tabla.

No obstante, pese a la significatividad de estos parámetros, cabe destacar el elevado valor de significatividad de los resultados obtenidos .000 tanto para los criterios goles

\begin{tabular}{|c|c|c|c|}
\hline Equipo & Goles en contra & $\begin{array}{c}\text { Clasificación goles en } \\
\text { contra }\end{array}$ & $\begin{array}{l}\text { Clasificación } \\
\text { final }\end{array}$ \\
\hline Progreso & 243 & 1 & 14 \\
\hline Yamal & 221 & 2 & 13 \\
\hline SpartakMoscow & 218 & 3 & 12 \\
\hline $\begin{array}{l}\text { Politech } \\
\text { Petersburg }\end{array}$ & 183 & 4 & 11 \\
\hline Partido Comunista & 162 & 5 & 10 \\
\hline Mytischi & 151 & 6 & 9 \\
\hline Norilsk Nickel & 148 & 7 & 7 \\
\hline Nueva Generación & 147 & 8 & 8 \\
\hline Tyumen & 143 & 9 & 6 \\
\hline Dinah & 139 & 10 & 3 \\
\hline Dynamo & 135 & 11 & 1 \\
\hline Siberiano & 134 & 12 & 4 \\
\hline Sinara & 110 & 13 & 5 \\
\hline Gazprom-Yugra & 96 & 14 & 2 \\
\hline
\end{tabular}


encajados como para la diferencia de goles. En el primer caso la relación es directa con valor .626, lo que quiere decir que cuanto mayor sea el número de goles en contra más baja será la clasificación en la liga, como se puede observar en la tabla 5 de la liga rusa, en la que se ordena de mayor a menor los goles en contra y la posición del equipo al final de liga, y en la que los últimos 6 se ordenan sistemáticamente siguiendo este criterio.

En cuanto a la diferencia de goles la relación es indirecta con valor -.912, cuanto mayor sea la diferencia de goles (a favor de los goles anotados) mejor será el puesto en la clasificación. Álvarez (2011) en su estudio del rendimiento deportivo de un equipo de futsal durante toda la temporada establece que es más importante la relación entre goles anotados-encajados encontrando diferencias significativas (.029), que el número de goles anotados. En la tabla 6 se muestran los resultados de los dos primeros y últimos clasificados de cada liga.

Tabla 6
\begin{tabular}{cccccc} 
Diferencia de goles y clasificación final por liga. \\
\multicolumn{2}{c}{ Rusia } & \multicolumn{2}{c}{ Italia } & \multicolumn{2}{c}{ España } \\
\hline Dif. Goles & Clasificación & Dif. Goles & Clasificación & Dif. Goles & Clasificación \\
\hline 112 & $2^{\circ}$ & 23 & $1^{\circ}$ & 91 & $1^{\circ}$ \\
86 & $1^{\circ}$ & 23 & $3^{\circ}$ & 78 & $2^{\circ}$ \\
-96 & $13^{\circ}$ & -29 & $11^{\circ}$ & -119 & $16^{\circ}$ \\
-135 & $14^{\circ}$ & -31 & $10^{\circ}$ & -133 & $15^{\circ}$ \\
\hline
\end{tabular}

Estos resultados indican que, atendiendo a criterios de significatividad, sería más apropiado el uso de la diferencia de goles o goles encajados frente a los goles anotados.

Así pues, con los datos detallados hasta el momento, se puede afirmar que el gol es la unidad de juego más trascendente para el objetivo final de cualquier equipo y por ello, se necesitan más estudios de ligas profesionales que relacionen el gol con otras variables como el momento, tipo de jugada, lugar de finalización, ventaja de jugar en casa, etc.

\section{Aplicación práctica}

Los datos recogidos de la competición permiten comprender mejor la realidad actual del fútbol sala europeo, pudiendo proporcionar retroalimentación a los jugadores y entrenadores, lo que les permite elaborar sus entrenamientos siguiendo la realidad competitiva y mejorar su rendimiento e interpretación de la actividad más allá de lo que se puede lograr mediante la observación personal.

\section{Limitaciones del estudio}

Se ha de tener presente la dificultad de la discusión por ser pocos los estudios sobre futsal y el gol, y de los existentes hay que tener en cuenta el uso de diferentes tipos de metodologías y muestras, como diferentes tipos de Campeonatos de duración larga o corta que modifica la forma y el estilo de juego y por consiguiente la forma de conseguir los goles.

\section{Conclusiones}

1. No se han encontrado diferencias significativas entre la capacidad goleadora de las diferentes ligas estudiadas, siendo la liga rusa la más goleadora con una media por partido de 8.16 goles seguida por la española con 7.37 goles y la italiana con 6.67 goles.

2. Los equipos locales anotan el $54.2 \%$ de los goles por el $45.8 \%$ como visitante. No se han encontrado diferencias significativas entre las ligas.

3. El gol es el mejor indicador del rendimiento y de la clasificación final. Existe relación entre los goles y la clasificación final, encontrando diferencias significativas tanto en los goles anotados como en los recibidos y su diferencia, siendo en estas dos últimas de .000 por lo que serán mejores predictores de la clasificación final que únicamente los goles anotados.

\section{Referencias}

Abdel-Hakim, H. (2014). Quantitative analysis of performance indicators of goals scored in the futsal World Cup Thailand 2012. Pamukkale Journal of Sport Sciences, 5(1), 113-127.

Agnew, G. A., \& Carron, A.V. (1994). Crow deffects and the home advantage and the home advantage. International Journal of Sports Psychology, 25, 53-62.

Agras, H., Ferragut, C., \& Abraldes, J.A. (2016). Match analysis infutsal: a systematic review. International Journal of Performance Analysis in Sport, 16(2), 652686.

Álvarez, J. (2011). Rendimiento de nuestro equipo. Estudio del rendimiento deportivo de un equipo de fútbol-sala durante toda la temporada (pp 53-59). En: El camino hacia el alto rendimiento deportivo en el fútbol sala. Asociación Nacional de Entrenadores de fútbol sala. España. ISBN 978-84-938302-8-1.

Álvarez, J., Murillo, V., \& García, A. (2018a). Influencia de la modificación del reglamento en la consecución de los goles en el fútbol sala. Revista Internacional de Medicina y Ciencias de la Actividad Física y el Deporte., 18(70), 213-216.

Álvarez, J., Murillo, V., García,A., \& Parra,A. (2018b). Análisis observacional de los goles de dos temporadas de la LNFS. Revista Internacional de Medicina y Ciencias de la Actividad Física y el Deporte., 18(69), 27-42.

Álvarez, J., Puente, J., Manero, J., \& Manonelles, P. (2004). Análisis de las acciones ofensivas que acaban en gol de la liga profesional de fútbol-sala española. Revista de Entrenamiento Deportivo, 18(4), 27-32.

Argudo-Iturriaga, F. M., Garcia-Cervantes, L., \& Ruiz-Lara, E. (2016). Factores asociados a la eficacia de gol en waterpolo. Factors associated with shooting efficacy in water polo. Retos, 29, 105-108.

Armatas, V., \& Yiannakos, A. (2008). Analysis and evaluation of goals scored in 2006 World Cup. Journal of Sport and Health Research, 2, 119-128.

Armatas, V., Yianakos, A., \& Sileloglou, P. (2007). Relationship between time and goal scoring in soccer games: Analysis of three World Cups. International Journal of Performance Analysis in Sport, 7(2), 48-58.

Ballesta-Castells, C., García-Romero, J., Fernández-García, J.C., \& Alvero-Cruz, J.R. (2015). Métodos actuales de análisis del partido de fútbol. Revista Internacional de Medicina y Ciencias de la Actividad Física y el Deporte, 15(60), 785-803. 
Beiztegui-Casado, C., Oliver-Coronado, J., \& Sosa-González, P.I. (en prensa) Portero-jugador en situaciones de inferioridad numérica ofensiva en balonmano: ¿penalización o ventaja? Goalkeeper-Field Player in Situations of Offensive Numerical Inferiority in Handball: Penalty or Advantage? Revista Internacional de Medicina y Ciencias de la Actividad Física y el Deporte.

Cachón, J., Campoy, T., Linares, D., Rodrigo, M., \& Zagalaz, M. L. (2012). Análisis cualitativo del cambio de las reglas de juego del fútbol sala (2006) en España. Ágora para la Educación Física y el Deporte, 14(3), 332-347.

Cachón, J., Valdivia, P., Lara,A., Zagalaz, M. L., \& Berdejo, D. (2014). Questionnaire: Loss of Entertainment in Spanish Futsal (PEFSE) - Results Analysis. American Journal of Sports Science and Medicine, 2(3), 83-87.

Campos, F., Pellegrinotti, I., Pasquarelli, B., Rabelo, F., Santa Cruz, R., \& Gómez, M.A. (2015). Effects of game-location and quality of opposition in futsal league. International Journal of Performance Analysis in Sport, 15, 598-607.

Carré, J., Muir, C., Belanger, J., \& Putnam, S. K. (2006). Precompetition hormonal and psychological levels of elite hockey players: Relationship to the «home advantage». Physiology \& Behavior, 89, 392-398.

Carrero-Pajares, I., Fernández-Echeverría, C., González-Silva, J., Conejero-Suárez, M., \& Moreno-Arroyo, M. P. (2017). Estudio predictivo de la eficacia de la recepción en voleibol juvenil masculino. Retos, 32, 214-218.

Casal, C.A., Losada, J.L., Maneiro, R., \& Ardá, T. (2017) Influencia táctica del resultado parcial en los saques de esquina en fútbol. Revista Internacional de Medicina y Ciencias de la Actividad Física y el Deporte, 17(68), 715-728.

Cassita, M. (2015). Analysis of goals second phase, futsal league 2013. Revista Brasileira de Futsal e Futebol, 7(2), 153-157.

Da Cuna, R., Goncalves, C., \& Eloi, P. (2016). Theorigin of Futsal 2014 League goals. Revista Brasileira de Futsal e Futebol, 8(29), 155-160.

De Bortoli, A. L., De Bortoli, R., \& Márquez, S. (2001). Utilización de coeficientes ofensivos para el análisis del rendimiento deportivo en el fútbol sala. Revista motricidad, 7, 7-17.

Estadísticas FIFA. Recuperado de: https://es.fifa.com/fifatournaments/statistics-and-records/futsalworldcup/ index.html

García, J.A., Menayo, R., \& Sánchez, J. (2015). Efectos de la práctica variable sobre el golpeo a portería en fútbol. Revista Internacional de Medicina y Ciencias de la Actividad Física y el Deporte, 15(60). 663-675.

García-Marín, P., Argudo-Iturriaga, F. M., \& Alonso-Roque, J. I. (2015). La acción de juego en desigualdad numérica por periodos en waterpolo. Retos, 27, 14-18.

Gea-García, G.M., \& Molina-Martín, J.J. (2015) Relación entre el nivel de juego y la ejecución del saque en voley playa femenino. Revista Internacional de Medicina y Ciencias de la Actividad Física y el Deporte, 15(59), 433-448.

Gil-Arias, A., Claver-Rabaz, F., Fernández-Echeverría, C., Moreno-Domínguez, A., \& Moreno-Arroyo, M.P. (2016) Análisis comparativo del saque en voleibol entre los campeonatos de España 2005-2010. Revista
Internacional de Medicina y Ciencias de la Actividad Física y el Deporte, 16(62), 439-456.

Gómez, M. A., Méndez, C., Indaburu, A., \& Travassos, B. (2018). Goal effectiveness after players' dismissals in professional futsal teams. Journal of Sports Sciences. 1(12), 335-342.

González-Silva, J., Moreno-Domínguez, A., FernándezEcheverría, C., Claver-Rabaz, F., \& Moreno-Arroyo, M. P. (2016). Asociación entre variables de la recepción y la zona de envío de la colocación en voleibol, en etapas de formación. Retos, 29, 149-152.

Gónzález-Silva, J., Moreno-Domínguez, A., FernándezEcheverría, C., Conejero-Suarez, M., \& Moreno-Arroyo, M. P. (2016). Características de la colocación en voleibol, en etapas de formación, en sets ganados y perdidos. Retos, 30, 43-47.

González-Víllora, S., García-López, L.M., \& Contreras-Jordán, O.R. (2015). Evolución de la toma de decisiones y la habilidad técnica en fútbol. Revista Internacional de Medicina y Ciencias de la Actividad Física y el Deporte, 15(59), 467-487.

Göral, K. (2018). Analysis of Serbia UEFA Futsal Euro 2016 Competitions in Terms of Some Variables. Journal of Education and Training Studies, 6(10), 445-454.

Goumas, C. (2004). Home advantage in Australian soccer. Journal of Science and Medicine in Sport, 17(1), 119123.

Grela, I. (2012). Análisis evolutivo de los goles en la primera división (LNFS) desde la temporada 2003-2004 hasta la 2012-2013. Revista Fútbol Táctico, 154.

Grela, I. (2013). Analysis of the goals in season 2012-2013 in the first división (LNFS) depending on the time and the match and result. Revista fútbol táctico, 78, 177-183.

Giani, G., Faria, G, \& Aparecida da Silva, S. (2018). Análise dos parâmetros técnico-tático dos gols da liga espanhola de futsal 2015/2016. Revista Brasileira de Futsal e Futebo, 10(36), 69-76.

Gutierrez, J., Castellano, J., Casamichana, D., \& SánchezSanchez, J. (2018). Influencia del tamaño del campo y horario del partido en la respuesta física de equipos de la Segunda División Española de Fútbol. Retos, 33, 213216.

Iglesias-Pérez, M. C., García-Ordóñez, E., \& Touriño-González, C. (2018). Game-Related Statistics in the Spanish Water Polo League: Differences between Seasons. Retos, 33, 228-232.

Jamieson, J. P. (2010). The home field advantage in athletics: A meta-analysis. Journal of Applied Social Psychology, 40(7), 1819-1848.

Jimenez-Olmedo, J. M., Espina-Agullo, J. J., \& Manchado, C. (2017). Análisis histórico de la efectividad de los lanzamientos a portería de balonmano masculino. Retos, 32, 228-232.

Jones, P. D., James, N. \& Mellalieu, S. D. (2004). Possession as a Performance Indicator in Soccer. International Journal of Performance Analysis in Sport, 4(1), 98-102.

Kacem, N., Guemri, A., Naffeti, C., \& Elloumi, A. (2016). Mechanism of Social Reproduction of the Culture Futsal: Modelling of the Universals of Futsal and Sense of the Rules of the Game: Analysis of Shooting at the European 
Cup Matches. Advances in Physical Education, 6, 5966.

Lago, C., Cancela, J. M., López, M. D. P., Fernández, F., \& Veiga, J. (2006). Evaluation of offensive actions in football performance against indicators of success in intensive diachronic retrospective designs. Apunts: Educación física y deportes, 12(72), 96-103.

Lapresa, D., Álvarez, L., Arana, J., Garzón, B., \& Caballero, V. (2013). Observational analysis of the offensive sequences that ended in a shot by the winning team of the 2010 UEFA Futsal Championship. Journal of Sports Sciences, 31(15), 1731-1739.

Lapresa, D., Álvarez, L., Arana, J., Garzón, B., \& Caballero, V. (2013). Observational analysis of the offensive sequences that ended in a shot by the winning team of the 2010 UEFA Futsal Championship. Journal of Sports Sciences, 31(15), 1731-1739.

López-Bondia, I., González-Rodenas, J., Calabuig-Moreno, F., Pérez-Turpin, J. A., \& Aranda-Malavés, R. (2017). Creating goal scoring opportunities in elite soccer. Tactical differences between Real Madrid CF and FCBarcelona. Retos, 32, 233-237.

López-Chamorro, J. M., Sánchez-Oliva, D., Pulido-González, J. J., \& Amado-Alonso, D. (2016). ¿Jugaré en la élite? Expectativas de llegar a profesional y su relación con la motivación en jóvenes jugadores de fútbol. Retos, 29, 153-156.

López-García, S., Maneiro-Dios, R., Ardá-Suárez, A., RialBoubeta, A., Losada-López, J.L., \& Casal-Sanjurjo, C.A. (2018) Tiros libres indirectos en fútbol de alto nivel. Identificación de variables explicativas. Revista Internacional de Medicina y Ciencias de la Actividad Física y el Deporte, 18 (70), 247-268.

Martínez, J.A. (2014). La influencia del primer cuarto en el resultado final en baloncesto. Revista Internacional de Medicina y Ciencias de la Actividad Física y el Deporte, 14(56), 755-769.

Martínez-Martínez, F. D., \& González-García, H. (2019). Efecto de marcar primero y la localización del partido en las principales ligas del fútbol europeo. Retos, 35, 242-245.

Massardi, F. P., Oliveira, M. C., \& Navarro, A. C. (2011). A incidência de gols na liga de futsalfeminina nos anos 2010 e 2011. Revista Brasileira de Futsal e Futebol, 3(9), 229-235.

Méndez-Dominguez, C., Gómez-Ruano, M.A., Ruiz-Pérez, L.M., \& Cul, Y. (2017). Unfavorable critical moments and way of facing them from the futsal coach's point of view through ad hoc questionnaire. RICYDE. Revista internacional de ciencias del deporte, 50(13), 339-355.

Moore, R., Bullough, S., Goldsmith, S., \& Edmondson, L. (2014). A Systematic review of Futsal Literature. American Journal of Sport Science and Medicine, 2(3), 108-116.

Nevill, A. M., \& Holder, R. L. (1999a). Home advantage in sport: an overview of studies on the advantage of playing at home. Sports Medicine, 28, 221-236.

Nevill, A., Balmer, N., \& Williams, M. (1999b). Crowd influence on decisions in association football. Lancet, 353, 141.

Pascual-Verdú, N., Álvarez-Amorós, A., Carbonell-Martínez, J. A., \& Pérez-Turpin, J. A. (2019). Análisis de patrón gol en competición de fútbol sala: $1^{\text {a }}$ División y $2^{\text {a }}$ División B. Retos, 35, 364-368.
Pérez-Ferreirós, A., Kalen, A., \& Rey, E. (2018). Short- and mid-term effects of the 2010 rule changes on game-related statistics in European basketball championships: An interrupted time series analysis. International Journal of Sports Science \& Coaching, 13(6), 1081-1089.

Piñar-López, M.I., Estévez-López, F., Ortega-Martín, V., Conde-González, J., Alarcón-López, F., \& Cárdenas-Vélez, D. (2014). Características de las fases de ataque en categoría infantil masculina. Revista Internacional de Medicina y Ciencias de la Actividad Física y el Deporte, 14(54), 265-278.

Rentero, L., João, P.V., \& Moreno, M.P. (2015). Análisis de la influencia del líbero en diferentes fases del juego en voleibol. Revista Internacional de Medicina y Ciencias de la Actividad Física y el Deporte, 15(60), 739-756.

Saavedra, J. M., Porgeirsson, S., Kristjánsdóttir, H., Chang, M, \& Halldórsson, K. (2017). Handball game-related statistics in men at Olympic Games (2004-2016): Differences and discriminatory power. Retos, 32, 260-263.

Saavedra-García, M., Gutiérrez-Aguilar, O., FernándezRomero, J.J. \& San Marques, P. (2015). Ventaja de jugar en casa en el fútbol español (1928-2011). Revista Internacional de Medicina y Ciencias de la Actividad Física y el Deporte, 15(57), 181-194.

Sabio-Lago, Y., Guerra-Balic, M., Cabedo-Sanromà, J., SolàSantesmases, J., \& Argudo-Iturriaga, F. (2018). Diseño, validación y fiabilidad de un instrumento para analizar acciones técnico-tácticas en waterpolo. Retos, 34, 57-65.

Sampedro, J., \& Prieto, J. (2011). La territorialidad como factor asociado a la ventaja de jugar en casa. Un estudio comparativo por regiones en la liga de fútbol y en la liga de fútbol sala de España. Motricidad European Journal of Human Movement, 26, 93-104.

Santana, W.C., Laudari, B. A., Istchuk, L.L., \& Arruda, F. M. (2013). Análise dos golsemjogos de futsalfeminino de alto rendimento. Revista Brasileira Ciência e Movimento. 21(24), 157-165.

Sarmento, H., Bradley, P, Anguera, M. T., Polido, T., Resende, R, \& Campanico, J. (2016). Quantifying the offensive sequences that result in goals in elite futsal matches. Journal of Sport Sciences, 34(7), 621-629.

Schneider, I., Da Cunha-Voser, R., Gomes-Voser, P. (2015). Analysis of goals scored and suffered by the under-17 futsal team from Nova Itaberaba-SC during the Santa Cataria championship 2013-2014. Revista Brasileira de Futsal e Futbol, 7(25), 327-331.

Sutter, M., \& Kocher, M. G. (2004). Favoritism of agents-the case of referees' home bias. Journal of Economic Psychology, 25(4), 461-469.

Trabal-Tañá, G., Daza, G., \& Riera, J. (2019). Habilidades técnicas del portero de hockey patines en la falta directa. Retos, 36, 69-73.

Vizcaíno, C., Sáenz-López, P., \& Rebollo, J.A. (2016). Relación de las reglas con los medios técnico-tácticos en minibasket. Revista Internacional de Medicina y Ciencias de la Actividad Física y el Deporte, 16(64), 807-823.

Wallace, H. M., Baumeister, R. F., \& Vohs, K. D. (2005). Audience support and choking under pressure: A home disadvantage?. Journal of Sports Sciences, 23(4), 429438. 